Dossier: La escritura en los desplazamientos: viajes, expediciones y naufragios

\title{
Viajes reales e imaginarios de Hernán Cortés en la corte del emperador Moctezuma II
}

\author{
A. Robert Lauer \\ The University of Oklahoma, Unites States \\ arlauer@ou.edu \\ https://orcid.org/0000-0001-9326-6200
}

Recibido: 14 de febrero de 2020

Aceptado: 30 de marzo de 2020

Resumen: El viaje de Hernán Cortés a la corte mexica del emperador Moctezuma II, aunque históricamente real, se modifica cada vez que es recontado. Tres versiones de este viaje se analizan en este estudio: la primera es la narrada en la Historia de la conquista de México, población y progresos de la América Septentrional, conocida por el nombre de Nueva España (Madrid, 1684), una obra panegírica de Antonio de Solís y Rivadeneira; la segunda es la cantada en el dramma per musica de Antonio Vivaldi intitulado Montezuma (Venecia, 1733), una 'comedia'; la tercera, también sustentada en Solís, es la representada en la tragedia per musica llamada Montezuma (Berlín, 1755), del compositor Carl Heinrich Graun y el estadista-y libretista-Friedrich II (der Große), de la Casa de Hohenzollern. Tenemos pues tres visiones radicalmente diferentes, aunque aceptables, del viaje cortesiano en tierras americanas: una encomiástica, una trágica y otra consoladora.

Palabras clave: Moctezuma II; Hernán Cortés; Antonio de Solís y Rivadeneira; Antonio Vivaldi; Carl Heinrich Graun; ópera; dramma per musica.

\section{Real and Imaginary Voyages of Hernán Cortés at the Court of Emperor Moctezuma II}

\section{cc) (i) (2)}

La Revista Estudios es editada por la Universidad de Costa Rica y se distribuye bajo una Licencia Creative Commons Atribución-NoComercial-Compartirlgual 3.0 Costa Rica. Para más información envíe un mensaje a 
Dossier: La escritura en los desplazamientos: viajes, expediciones y naufragios

Abstract: The voyage of Hernán Cortés to the Mexica court of Emperor Moctezuma II, although historically real, varies every time it is re-told. Three versions of this voyage are analyzed in this study: the first one is the one narrated in the History of the Conquest of Mexico by the Spaniards (Madrid, 1684), a panegyric written by Antonio Solís y Rivadeneira; the second is the one sung in the dramma per musica of Antonio Vivaldi called Montezuma (Venice, 1733), a 'comedy'; the third one, also based on Solis' History, is the one represented in the tragedia per musica named Montezuma (Berlin, 1755), composed by Carl Heinrich Graun and Friedrich II (der Große) of the House of Hohenzollern. Hence, we have three radically different but acceptable visions of Cortés' voyage in the Americas: an encomiastic one, a tragic one, and a consolatory one.

Keywords: Moctezuma II; Hernán Cortés; Antonio de Solís y Rivadeneira; Antonio Vivaldi; Carl Heinrich Graun; opera; dramma per musica.

En este trabajo se plantea la importancia de tres versiones de un viaje de Hernán Cortés, fundador del México post-indígena, a la corte del emperador Moctezuma II de Tenochtitlán'. La primera es histórica y se basa en la Historia de la conquista de México, población y progresos de la América Septentrional, conocida por el nombre de Nueva España (Madrid, 1684), de Antonio de Solís y Rivadeneira. La segunda, basada en el texto de Solís, es el dramma per musica de Antonio Vivaldi intitulado Montezuma (Venecia, 1733), una 'comedia'. La tercera, de asunto trágico, también sustentada en Solís, es la tragedia per musica llamada Montezuma (Berlín, 1755), del compositor Carl Heinrich Graun y el estadista-y libretistaFriedrich II (der Große), de la Casa de Hohenzollern. Los desenlaces, similares y desemejantes a la vez, poetizan un hecho históricamente impactante en el imaginario colectivo para crear diversos textos alternativos: uno, el de Solís y Rivadeneira, de tono epidíctico; otro, el de Vivaldi, de matiz triunfante; y el tercero, de Graun y Friedrich II, de tinte crítico. El percibido viaje de Hernán Cortés a América, así como su conjeturada despedida, es, por tanto, una estética-literaria y musical- reconstrucción demostrativa, quimérica y comprometida. Se evidencia así el dictamen de Friedrich Nietzsche de que no hay hechos; solo interpretacionesii.

\section{(c) (i) (-)}

La Revista Estudios es editada por la Universidad de Costa Rica y se distribuye bajo una Licencia Creative Commons Atribución-NoComercial-CompartirIgual 3.0 Costa Rica. Para más información envíe un mensaje a revistaestudios.eeg@ucr.ac.cr. 
Dossier: La escritura en los desplazamientos: viajes, expediciones y naufragios

Empezamos con la Historia de la conquista de México, población y progresos de la América Septentrional, conocida por el nombre de Nueva España de Antonio de Solís y Rivadeneira, obra que dio origen a múltiples piezas literarias y musicales a lo largo del siglo XVIII en Europa. Aunque el texto de Solís está basado en hechos históricos relacionados con la conquista de Méjico, el énfasis del relato cae en la figura del conquistador, Hernán Cortés de Monroy y Pizarro Altamirano. Su nombre es el más citado a lo largo de esta memoriaiii, seguido después por el del emperador

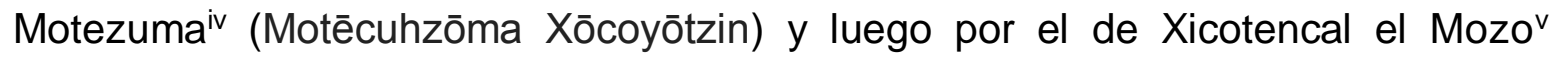
(Xīcohténcatl Āxāyacatzin), el famoso guerrero tlaxcalteca que después de combatir contra los españoles se alió a ellos en la conquista de Tenochtitlán.

Desde el capítulo nueve del primer libro hasta el 25 del quinto, Cortés domina la relación de Solís. Es significativo que a mediados del noveno capítulo de la primera parte, Solís interrumpa la narrativa para ofrecer una digresión sobre el protagonista del relato: "Pero antes que pasemos adelante, será bien que digamos quién era Hernán Cortés" (Solís y Rivadeneira, versión de 1997, p. 41 [1.9]). Lo que prosigue es un encomio del héroe que sigue las pautas de un modélico discurso panegírico, como se vería, v. gr., en Menandro de Laodicea, conocido como 'el Rétor'. Atribuye, pues, Solís la conquista de la Nueva España a dos factores: el valor y el entendimiento de Cortés. Alude de esta forma al tópico clásico de 'sapientia et fortitudo', así como al de la felicidad del destino del héroe que, según Solís, dispone Dios "con la elección del hombre" (Solís y Rivadeneira, versión de 1997, p. 41 [1.9]). Extraordinariamente, Solís, en una continua y extensa alocución, usa el exordio sobre el conquistador, el cual debiera iniciar un discurso, simultáneamente como peroración, indicando de esta manera un inicio y un próspero fin. Se precluye así cualquier tipo de suspenso o dubitación en el lector respecto al fin del héroe o de la narrativa. Lo que se relatará a lo largo de los cinco libros siguientes será simplemente la evidencia o confirmación de los hechos. De esta forma, el leedor se enfocará en el 'cómo' de la narración y no tanto en el 'qué' de la misma. El texto

\section{(ब) $(\Theta \odot$}

La Revista Estudios es editada por la Universidad de Costa Rica y se distribuye bajo una Licencia Creative Commons Atribución-NoComercial-CompartirIgual 3.0 Costa Rica. Para más información envíe un mensaje a revistaestudios.eeg@ucr.ac.cr. 
Dossier: La escritura en los desplazamientos: viajes, expediciones y naufragios invita al análisis, no solo a la anunciación de hechos que en pleno 1684 habrían sido conocidos. Es, etimológicamente, una historia > historein > indagación, juicio, y no solamente un acto de ver > idein o de conocimiento > eidenai.

Lo que sigue en el discurso son las partes encomiásticas que se evidenciarían en un panegírico. Se menciona el lugar de nacimiento de Cortés, Medellín, villa de Extremadura, así como el nombre de sus padres, Martín Cortés de Monroy y doña Catalina Pizarro Altamirano, "cuyos apellidos no sólo dicen, sino encarecen lo ilustre de su sangre" (Solís y Rivadeneira, versión de 1997, p. 41 [1.9]). De esta forma, Solís seguiría la recomendación de Menandro el Rétor en su tratado sobre el "Basilikos logos"(Menandro el Rétor, trad. en 2019, pp. 139-163 [368-377]): mencionar, si son ilustres, después del proemio de una epideixis o demostración, primero el lugar y después la familia del héroe ${ }^{\text {vi }}$. Subsiguientemente, Menandro recomienda indicar el nacimiento, la naturaleza, la crianza o la educación del héroe vii. Solís en efecto alude a dos de estos elementos al mencionar que Cortés "Diose a las letras en su primera edad [educación], y cursó en Salamanca dos años, que le bastaron para conocer que iba contra su natural [naturaleza], y que no convenía con la viveza de su espíritu aquella diligencia perezosa de los estudios. Volvió a su casa resuelto a seguir la guerra" (Solís y Rivadeneira, versión de 1997, p. 41 [1.9]). Se reitera así el tema de 'sapientia et fortitudo' ya señalado en el exordio.

En consecuencia, Menandro recomienda hacer mención de las virtudes y acciones del héroe viii. Se nota la combinación de ambas en Solís cuando menciona que Cortés, después de un accidente que impide su participación en Italia de la guerra que "era la de más pundonor" (Solís y Rivadeneira, versión de 1997, p. 41 [1.9]), "Inclinóse a pasar a las Indias, que como entonces duraba su conquista, se apetecían con el valor más que con la codicia" (Solís y Rivadeneira, versión de 1997, p. 41 [1.9]). Al llegar a Santo Domingo, Cortés "halló grande agasajo y estimación en todos, y tan agradable acogida en el gobernador [don Nicolás de Ovando], que le admitió desde luego entre los suyos" (Solís y Rivadeneira, versión de 1997, p. 41

\section{(C) $(0 \bigcirc)$}

La Revista Estudios es editada por la Universidad de Costa Rica y se distribuye bajo una Licencia Creative Commons Atribución-NoComercial-CompartirIgual 3.0 Costa Rica. Para más información envíe un mensaje a revistaestudios.eeg@ucr.ac.cr. 
Dossier: La escritura en los desplazamientos: viajes, expediciones y naufragios [1.9]). No acostumbrado a la ociosidad, pidió licencia para partir a Cuba, donde "Consiguió brevemente la opinión de valeroso, y tardó poco más en darse a conocer su entendimiento" (Solís y Rivadeneira, versión de 1997, p. 41 [1.9]). Nótese de nuevo la repetición del tópico de 'sapientia et fortitudo'.

La apariencia física es otro importante factor que se menciona en los encomios, como señala Cicerón en De oratore (trad. de 1988, pp. 230-231 [2.10.4546] $)^{\mathrm{ix}}$. Los valores atribuidos a la fortuna en el caso de un hombre agraciado serían, entre ellos, la etnia, la riqueza, las conexiones, las amistades, el poder, la salud, la belleza, el vigor, los talentos y los atributos físicos externos. La gentil apariencia en el hombre, en combinación con la virtud, es consiguientemente un providencial signo, como comenta en su Retórica Aristóteles. Por ende, varios componentes de la prosperidad humana en el hombre serían la noble crianza, la fuerza física, la estatura, la habilidad atlética, la buena reputación, la bienaventuranza, la autoridad y la influencia que se ejerce en otros individuos. Solís por su parte señala que Hernán Cortés "Era mozo de gentil presencia y agradable rostro; y sobre estas recomendaciones comunes de la naturaleza, tenía otras de su propio natural que lo hacían amable" (Solís y Rivadeneira, versión de 1997, p. 41 [1.9]). Cortés, según Solís, también era festivo y discreto en las conversaciones y generoso con sus amigos, quienes agradecían sus favores. También, como recomendaría Menandro, Hernán Cortés casó bien con doña Catalina Suárez Pacheco, "doncella noble y recatada" (Solís y Rivadeneira, versión de 1997, p. 41 [1.9]), y recibió de Diego Velázquez, gobernador de la isla de Cuba, un repartimiento de indios y la vara de alcalde en la villa de Santiago. Aun en este caso, Solís parece seguir a Menandro al indicar éste que el héroe suele recibir esclavos, aunque en este caso serían vasallos de la corona, a quienes administrar. Dando indicios de su capacidad y afabilidad, Cortés, recomendado por el contador Amador de Lariz y el secretario Andrés de Duero, es nombrado, por el propio Diego Velázquez, "'capitán general de

\section{(๑) $\odot \odot$}

La Revista Estudios es editada por la Universidad de Costa Rica y se distribuye bajo una Licencia Creative Commons Atribución-NoComercial-CompartirIgual 3.0 Costa Rica. Para más información envíe un mensaje a revistaestudios.eeg@ucr.ac.cr. 
Dossier: La escritura en los desplazamientos: viajes, expediciones y naufragios

la armada, y tierras descubiertas y que se descubriesen'” (Solís y Rivadeneira, versión de 1997, p. 42 [1.9]).

Los subsiguientes viajes continentales de Cortés en América servirán solo de confirmación de lo que el panegírico del noveno capítulo del primer libro ya había indicado. El héroe conquistador indudablemente tendrá sus altibajos. No obstante, con la posterior rendición de Guatimozín (Cuāuhtémōc), decimotercer tlatoani de los mexicas, termina, para Solís, la vida triunfante de Cortés, estableciéndose así, como él menciona, la gran monarquía de Nueva España. En la última peroración de Solís al final del capítulo 25 del quinto libro se expresa el deseo de que el César (Carlos V) otorgue al extremeño una corona "digna de sus reales sienes" (Solís y Rivadeneira, versión de 1997, p. 353 [5.25]). En un colofón se concluye el encomio en forma boyante y jaculatoria: “¡Admirable conquista, y muchas veces ilustre capitán! de aquellos que producen tarde los siglos, y tienen raros ejemplos en la historia" (Solís y Rivadeneira, versión de 1997, p. 353 [5.25]). Este elocuente y retórico remate encuentra un eco en el deseo que expresa Menandro el Rétor al final de su discurso sobre el "Basilikos logos": que el mando del estadista dure largo tiempo y que su potestad pase a sus descendientes (Menandro, trad. en 2019, p. 163 [377]).

La obra histórica de Solís, traducida a varios idiomas, entre ellos el inglés, el francés, el italiano, el alemán y el danés, inspiró a múltiples dramaturgos y musicólogos en España y el resto de Europa a lo largo del Siglo de las Luces. En esta ocasión nos enfocamos en las dos primeras óperas fundamentadas en la Historia de Solís: 1) Montezuma (1733), del veneciano Antonio Vivaldi, basada en el libreto de Girolamo Giusti; y 2) Montezuma (1755) del brandenburgués Carl Heinrich Graun, inspirada en el texto del rey de Prusia, Friedrich II de Hohenzollern. Ambas son opere serie: la primera un dramma per musica; la segunda una tragedia per musica. Por lo tanto, las dos obras siguen las convenciones que regulan el género según lo iniciara el poeta romano Pietro Antonio Domenico Bonaventura

\section{(๑) $\odot \odot$}

La Revista Estudios es editada por la Universidad de Costa Rica y se distribuye bajo una Licencia Creative Commons Atribución-NoComercial-CompartirIgual 3.0 Costa Rica. Para más información envíe un mensaje a revistaestudios.eeg@ucr.ac.cr. 
Dossier: La escritura en los desplazamientos: viajes, expediciones y naufragios

Trapassi (1698-1781), mejor conocido como Metastasio. La diferencia entre dramma y tragedia es de matiz. El contenido histórico o mitológico del dramma, así como el uso de la lengua italiana o alemana, diferencian las opere serie de las buffe, las cuales versan sobre asuntos cotidianos y estereotipos caricaturescos, empleándose asimismo dialectos locales. Otrosí, una opera seria no requiere ser necesariamente una tragedia; puede ser, en efecto, un drama con un fin feliz o una dramatización de finalidad infausta. Montezuma de Vivaldi es precisamente una commedia per musica, mientras que Montezuma de Graun es, dentro del mismo género de la opera seria, una tragedia per musica. La diferencia en ambos casos no es pues de contenido, el cual es histórico en las dos obras, sino de modalidad: próspera en la primera; en la segunda, ruinosa, pero no burlesca, ordinaria o buffa.

Estructuralmente, la ópera de Montezuma de Vivaldi consiste de una sinfonía inicial de tres movimientos (allegro, adagio molto y allegro) y tres actos: el primero de 16 escenas, el segundo de 14 y el tercero de 12. El tercer acto contiene a la vez una sinfonia funebre de 1:31 en la escena quinta y dos coros triunfantes en las escenas 11 y 12. Cada acto contiene varias arias (7 en el primero, 6 en el segundo y 6 en el tercero) y recitativos (10 en el primero, 7 en el segundo y 8 en el tercero). Siete de los recitativos (cinco en el primer acto y dos en el segundo) son recitativi accompagnati, asistidos de la orquesta. Estas serían las secciones más estructuradas y menos improvisadas de canto. Es de notar que Montezuma sobresale en este tipo de recitativo en el primer acto, donde canta solo tres veces (en 1.2, 1.8 y 1.15), mientras que Fernando Cortés canta solo de esta forma una única vez (en 2.4). Respecto a arias, Montezuma se vale de tres (1.2, 1.15 y 3.10), mientras que Fernando de cuatro (en 1.5, 1.14, 2.7 y 3.1). Se observa a la vez que, debido a su protagonismo, Ramiro (1.7, 2.10 y 3.2), supuesto hermano de Fernando, y Teutile (1.6, 2.13 y 3.5), hija de Montezuma y amante de Ramiro, cantan arias solos también en tres ocasiones. Otros cantantes que se valen de arias en menor

\section{(c) (1)(-)}

La Revista Estudios es editada por la Universidad de Costa Rica y se distribuye bajo una Licencia Creative Commons Atribución-NoComercial-CompartirIgual 3.0 Costa Rica. Para más información envíe un mensaje a revistaestudios.eeg@ucr.ac.cr. 
Dossier: La escritura en los desplazamientos: viajes, expediciones y naufragios escala serían Mitrena (1.16 y 2.14), la esposa de Montezuma, y el general mejicano Asprano (2.1, 3.3).

Dramáticamente, la ópera de Vivaldi empieza in extrema res en la ciudad de Tenochtitlán ${ }^{x i}$. Ha ocurrido una decisiva batalla entre españoles y mexicas y Fernando Cortés ha salido vencedor. En un bello recitativo acompañado, Montezuma expresa su dolor ante su rendimiento: "Son vinto eterni Dei! Tutto in un giorno lo splendor de'miei fasti, e l'alta Gloria del valor Messican cade svenata" (Giusti, versión de 1992, p. 90 [1.1])xii. A la vez, tanto su esposa Mitrene como su hija Teutile están dispuestas a morir en lugar de aceptar el supuesto cautiverio que impondría el triunfador:

MOT. [. . .] Corraggiosa in tanto prendi ... Questo ti servi (li dà un Coltello).

Di strommento à mostrar il tuo gran core, e pria ch'il traditore, stringa le destre di servil catena,

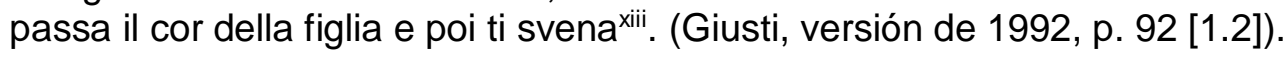

En una analepsis nos enteramos de que Montezuma ha roto un pacto anterior con Cortés, a quien posteriormente ataca y hiere. También sabemos que existe una relación sentimental entre Ramiro, hermano de Fernando, y Teutile, hija de Montezuma. Esta segunda intriga amorosa complica las lealtades de personajes secundarios, quienes casi desplazan dramática y musicalmente a los principales, Montezuma y Cortés, en la primera y primordial acción sobre la conquista de Méjico. Para establecer el orden, Fernando pone en prisión a Montezuma, acto que impulsa una reacción bélica de los mexicas y su general Asprano. Después de consultar los magos al dios Uccilibos (Huitzilopochtli), Asprano se entera de que "Teutile, ed un Ispano col Sacrificio loro l'Impero, e il Genitor salvar potrano"' (Giusti, versión de 1992, p. 136 [2.13]) ${ }^{\text {iv }}$. Mitrena y Montezuma piensan que deben sacrificar a Teutile y Fernando, mas tras otro encuentro bélico en que de nuevo triunfan las fuerzas europeas, el magnánimo Cortés le ofrece a Montezuma gobernar el imperio si se Commons Atribución-NoComercial-CompartirIgual 3.0 Costa Rica. Para más información envíe un mensaje a revistaestudios.eeg@ucr.ac.cr. 
Dossier: La escritura en los desplazamientos: viajes, expediciones y naufragios entrega a un nuevo Dios y a la Corona Española. Al acceder Montezuma a la petición de Cortés, se sella la nueva alianza entre españoles y mexicas, casándose Ramiro con Teutile. Se cumple así el oráculo del dios Uccilibos, quien había pronosticado un sacrificio entre Teutile y un español. Montezuma cierra así el drama, acompañado de un coro:

MOT. Ne vostri Dei gran verità si scorge cade il Messico è ver; ma poi risorge.

CORO. Imeneo, che sei d'amori dolce ardor, nodo immortale per la copia alma, e Reale Stringi l'alma, e annoda i coriv. (Giusti, versión de 1992, p. 158 [3.12]).

En este caso, el viaje de Cortés al imperio de los mexicas tiene, al principio, un fin funesto; al final una conclusión feliz que sugiere una despedida (otro viaje), dejándose en tierras mejicanas al hermano del conquistador y a la princesa indígena en lazos de Himeneo.

La ópera del libretista Federico Il y el compositor musical Carl Heinrich Graun, Montezuma, de 1755, a diferencia de Motezuma de Vivaldi, empieza no in extrema sino in medias res, en un jardín imperial, en el momento en que Montezuma está en su pleno poder y justo antes de la inminente llegada de Cortés a su imperio:

MONTEZUMA: [ . . .] il Messico è felice. Frutto di quella libertà, che, unita alla prudenza, al solo fren soggiace delle leggi, ch'io stesso sono il primo ad osservare; il popol mio di stabil Gaudio e bel riposo abbonda, e il mio poter su l'amor suo si fonda ${ }^{x i}$. (Tagliazucchi, trad. en 1992, pp. 62-64 [1.1]).

En su ingenuidad, Montezuma no oye la advertencia de Pilpatoè, el general imperial, quien le advierte que los 300 "stranieri erroi" (Tagliazucchi, trad. en 1992, p. 68 [1.3]) [“héroes extraños" (Reinshagen-Hernández, trad. en 1992, p. 69 [1.3])] que acaban de llegar a su imperio, "al lor coraggio, al lor fiero ardimento" (Tagliazucchi, trad. en 1992, p. 68; 1.2) [«con su valor y su fiereza» (Reinshagen-Hernández, trad. en 1992,

\section{(C) $(\otimes \otimes \odot$}

La Revista Estudios es editada por la Universidad de Costa Rica y se distribuye bajo una Licencia Creative Commons Atribución-NoComercial-CompartirIgual 3.0 Costa Rica. Para más información envíe un mensaje a 
Dossier: La escritura en los desplazamientos: viajes, expediciones y naufragios p. 69 [1.2])] serían capaces de todo. Tampoco advierte las palabras de Eupafórice, esposa del emperador y reina de Tlaxcala, respecto a aquel ejército de "straniero conquistador" (Tagliazucchi, trad. en 1992, p. 72 [1.4]) [“conquistadores extraños" (Reinshagen-Hernández, trad. en 1992, p. 73 [1.4])]. Aún ante la arrogancia de Narvès, capitán español de Ferdinando Cortés, ante el emperador, Montezuma declara que "Se pacifici sono, se vengono ad implorare i nostri auspici, vedran che Ior saremo ospiti amici" (Tagliazucchi, trad. en 1992, p. 78 [1.7]) [“Si vienen en paz, si vienen a solicitar nuestra protección, verán que los trataremos como amigos" (Reinshagen-Hernández, trad. en 1992, p. 79 [1.7])].

Cortés, no obstante, no viene como amigo sino como representante de un poder europeo: “In questo giorno, prima che l'ombre agli occhi de'mortali involino la luce, arbitri affatto del Messico saremo, e regnar vi faremo col nostro Re la nostra religione ancor" (Tagliazucchi, trad. en 1992, p. 88 [2.1]) [“Este mismo día, antes de que la noche de oscuros ojos rapte la luz del día, seremos dueños del México antiguo; imperará en esta rica tierra nuestro rey y con él, nuestra santa fe" (Reinshagen-Hernández, trad. en 1992, p. 89 [2.1])]. Cuando Pilpatoè y Tezeuco, chambelán de Montezuma, cuestionan por qué Cortés está formando a sus soldados para un perentorio encuentro militar, los europeos acometen a los americanos, quienes huyen al oír los disparos de las inauditas armas de fuego. Esta parte está acompañada de una breve (de 0:36 segundos) sinfonía guerrera de movimiento allegro. Subsiguientemente, Cortés indica que sus acciones tienen un objetivo ulterior: acabar con los bárbaros sacrificios humanos y cristianizar a los mexicas: "Più che di far conquiste, cerchiam di farvi noto il nostro Dio, e stabilir fra voi quella perfetta religión, che a questo Nume è accetta" (Tagliazucchi, trad. en 1992, p. 100 [2.7]) ["No por conquistar, no, sólo por nuestro Dios vamos a la batalla; convertirlos a ustedes, eso queremos, a esta santa pura fe que place a nuestro Dios" (Reinshagen-Hernández, trad. en 1992, p. 101 [2.7])]. Después de que Montezuma trata de matar a Cortés por su supuesta arrogancia, Cortés le advierte: "Pensa

\section{(๑) $\odot \odot$}

La Revista Estudios es editada por la Universidad de Costa Rica y se distribuye bajo una Licencia Creative Commons Atribución-NoComercial-CompartirIgual 3.0 Costa Rica. Para más información envíe un mensaje a revistaestudios.eeg@ucr.ac.cr. 
Dossier: La escritura en los desplazamientos: viajes, expediciones y naufragios prestar da saggio al mio monarca omaggio, e del tuo culto insano, lascia il fallace error. Meré, soccorso avrai, fidati pure a me; tutto sperar potrai dal mio clemente Re" (Tagliazucchi, trad. en 1992, p. 104 [2.7]) ["Sé prudente y obedece lealmente a tu nuevo monarca [Carlos V], abandona los falsos dioses, abandona todo error. Pronto la vida te volverá a sonreír, confía en mí. Todo puedes esperar de mi clemente señor" (Reinshagen-Hernández, trad. en 1992, p. 105 [2.7])]. No obstante, Montezuma trata de organizar un levantamiento contra los invasores; acto que Cortés ahora no perdonará. Sin embargo, en este momento, el conquistador le ofrece salvar su vida al emperador si éste abandona sus falsos dioses, hecho que Montezuma, como el Moctezuma histórico, se niega a hacerxvii. La ópera termina con la ejecución de Montezuma, llevada a cabo entre bastidores, y concluye con un conmiserativo coro de los vencidos:

CORO. Oh Cielo! Ahi giorno orribile, di delitti esecrabili. O terra, che li tolleri, apri le tue voragini. Fuggiam, fuggiam dai barbari, voi, giusti Dei, salvateci, movetevi a pietàxviii. (Tagliazucchi, trad. en 1992, p. 134 [3.5]).

El viaje de Cortés aquí, por ende, tiene funestas consecuencias para los mexicas.

Como podemos ver, el viaje de Cortés a América, aunque real, es a la vez arquetípico o imaginario, pues forma parte de un modelo simbólico común a la humanidad: el recorrido o la peregrinación. En el caso de Solís, el viaje de Cortés se asocia con la figura privilegiada del conquistador: es su destino encontrar un nuevo continente y ofrecérselo a su superior, el Césarix. El noveno capítulo del primer libro es pues un encomio al mismo que sirve para probar la tesis del historiador. En el caso de Graun y Federico II, el viaje inminente de Cortés, que se menciona en la ópera in medias res, se asocia con algo fatídico que culmina en la destrucción del imperio mexica. El agente es, pues, un actante de destrucción, una amenaza. La ópera, por ende, es una tragedia per musica. En el caso de Giusti y Vivaldi, el viaje de Cortés in extrema res se asocia inicialmente con algo nefasto y

\section{(c) () (2)(2)}

La Revista Estudios es editada por la Universidad de Costa Rica y se distribuye bajo una Licencia Creative Commons Atribución-NoComercial-CompartirIgual 3.0 Costa Rica. Para más información envíe un mensaje a revistaestudios.eeg@ucr.ac.cr. 
Dossier: La escritura en los desplazamientos: viajes, expediciones y naufragios final; no obstante, la sugerida despedida del conquistador al final de la ópera indica no el fin sino el principio de un nuevo imperio, un nuevo mundo, una nueva unión entre mexicas (Teutile) y españoles (Ramiro). Esto se expresa por medio de un dramma per musica. El viaje cortesiano, pues, aunque real, es una invención retórica en el caso de Solís, un acontecimiento trágico en el caso de Graun, y el principio de una nueva civilización en el caso de Vivaldi. Ese viaje real e imaginario, así llamado por haber pasado por varios filtros genéricos, es pues una invención que, aunque real, sirve otros propósitos en el imaginario colectivo: privilegiar a un héroe, explicar una destrucción y evidenciar un nuevo comienzo. El viaje cortesiano es pues real e imaginario a la vez: histórico, encomiástico, trágico y cómico (o serio). Todo será cuestión de perspectiva. Volvemos a las insignes palabras de Nietzsche, que servirán aquí de conclusión: no hay hechos, solo interpretaciones (Nietzsche, 1968, p. 458).

\section{Obras citadas}

Aristóteles (trad. en 2006). The "Art" of Rhetoric. J. H. Freese (ed. y trad.). Cambridge, MA, EUA \& Londres, Reino Unido: Harvard University Press. Loeb Classical Library 193.

Bartrina, F. (trad. en 1992 del libreto de G. Giusti al español). Montezuma, dramma per musica [CD]. A. Vivaldi (compositor), G. Giusti (libretista), J.-C. Malgoire (director musical). Région Norde / Pas-de-Calais, Francia: Auvidis Atelier Lyrique de Tourcoing.

Cicerón (trad. de 1988). De oratore. Libros I, II. Vol. 3. E. W. Sutton y H. Rackham (trad.). Cambridge, MA, EUA \& Londres, Reino Unido: Harvard University Press \& William Heinemann, 1988. Loeb Classical Library 348.

Friedrich II "der Große" (1992). Libreto, Montezuma [CD]. C. H. Graun (compositor), J. Goritzki (director musical), Kammerchor Cantica Nova, Deutsche Kammerakademie. Köln, Nordrhein-Westfalen, Alemania: Capriccio.$$
\text { (c) (1)(2) }
$$

La Revista Estudios es editada por la Universidad de Costa Rica y se distribuye bajo una Licencia Creative Commons Atribución-NoComercial-CompartirIgual 3.0 Costa Rica. Para más información envíe un mensaje a revistaestudios.eeg@ucr.ac.cr.
} 
Dossier: La escritura en los desplazamientos: viajes, expediciones y naufragios

Giusti, G. (1992) (libretista). Montezuma, dramma per musica [CD]. A. Vivaldi (compositor), J.-C. Malgoire (director musical). Région Norde / Pas-deCalais, Francia: Auvidis Atelier Lyrique de Tourcoing.

Menandro el Rétor (trad. en 2019). The Royal Oration (Basilikos logos). En J. Henderson (ed. y trad.). Treatise I/ (pp. 138-163). Cambridge, MA, EUA \& Oxford, Reino Unido: Harvard University Press. Loeb Classical Library 539. Montezuma, dramma per musica (1992 CD). A. Vivaldi (compositor), G. Giusti (libretista), F. Bartrina (trad. al español), J.-C. Malgoire (director musical).

Région Norde / Pas-de-Calais, Francia: Auvidis Atelier Lyrique de Tourcoing. Montezuma, Oper in 3 Akten (1992 CD). C. H. Graun (compositor), Friedrich II "der Große" y G. Tagliazucchi (libretistas), J. Goritzki (director musical), Kammerchor Cantica Nova, Deutsche Kammerakademie. Köln, NordrheinWestfalen, Alemania: Capriccio.

Nietzsche, F. (trad. en 1968). Notes (1888). En W. Kaufmann (ed. y trad.), The Portable Nietzsche (pp. 457-459). Nueva York, NY, EUA: The Viking Press.

Reinshagen-Hernández, O. (trad. del libreto al español en 1992). Montezuma, Oper in 3 Akten [CD]. C. H. Graun (compositor), Friedrich II (libretista) y G. Tagliazucchi (trad. del libreto al italiano), J. Goritzki (director musical), Kammerchor Cantica Nova, Deutsche Kammerakademie. Köln, NordrheinWestfalen, Alemania: Capriccio.

Solís y Rivadeneira, A. de (versión de 1997). Historia de la conquista de México, población y progresos de la América Septentrional conocida por el nombre de Nueva España. E. O'Gorman y J. Valero Silva (ed.). México, D. F., México: Editorial Porrúa, 1997.

Tagliazucchi, G. (trad. del libreto al italiano en 1992). Montezuma, Oper in 3 Akten [CD]. C. H. Graun (compositor), Friedrich II "der Grosse" (libretista), G. Tagliazucchi (trad. del libreto al italiano), O. Reinshagen-Hernández (trad. del libreto al español), J. Goritzki (director musical), Kammerchor Cantica Nova,

\section{(๑) $\Theta \odot$}

La Revista Estudios es editada por la Universidad de Costa Rica y se distribuye bajo una Licencia Creative Commons Atribución-NoComercial-CompartirIgual 3.0 Costa Rica. Para más información envíe un mensaje a revistaestudios.eeg@ucr.ac.cr. 
Dossier: La escritura en los desplazamientos: viajes, expediciones y naufragios

Deutsche Kammerakademie. Köln, Nordrhein-Westfalen, Alemania: Capriccio, 1992.

Vivaldi, A (1992). Montezuma, dramma per música [CD]. G. Giusti (libretista), F. Bartrina (trad. del libreto al español), J.-C. Malgoire (director musical). Région Norde / Pas-de-Calais, Francia: Auvidis Atelier Lyrique de Tourcoing, 1992.

Notas

' Un estudio más elaborado de la música de Montezuma de Carl Heinrich Graun, en relación a una obra literaria intitulada Motezuma (1784), de Bernardo María de Calzada, aparecerá próximamente en la revista Hipogrifo.

ii "Against that positivism which stops before phenomena, saying 'there are no facts,' I should say: no, it is precisely facts that do not exist, only interpretations. .." (Nietzsche, F. trad. en 1968, p. 458).

iii Véase el índice de Solís y Rivadeneira, versión de 1997, pp. 369-370, 375.

iv Véase el índice de Solís y Rivadeneira, versión de 1997, p. 373.

v Véase el índice de Solís y Rivadeneira, versión de 1997, p. 377.

vi "After these introductions move on to his homeland. Here you should take into consideration whether or not it is famous. If it is famous, you should comment on it first, even before you speak of his lineage" (Menandro el Rétor, trad. en 2019, pp. 141-142 [369]).

vii "After the topic of birth, say something about his nature [. . .] Next comes the section on upbringing [ . . . At this point, if you can find any similar examples, provide them, but if he does not have an illustrious rearing as Achilles had with Chiron, then you should look instead to his education" (Menandro el Rétor, trad. en 2019, p. 147 [371]).

\section{(๑) $\odot \odot$}

La Revista Estudios es editada por la Universidad de Costa Rica y se distribuye bajo una Licencia Creative Commons Atribución-NoComercial-CompartirIgual 3.0 Costa Rica. Para más información envíe un mensaje a revistaestudios.eeg@ucr.ac.cr. 
Dossier: La escritura en los desplazamientos: viajes, expediciones y naufragios

viii "His activities will have a place for exposition, activities being character traits apart from competitive deeds, for activities entail an indication of character." (Menandro el Rétor, trad. en 2019, p. 149 [372]).

ix " $[\mathrm{H}] \mathrm{e}$ who proposes to be the panegyrist of anyone will understand that he has in the first place to deal fully with the favours of fortune. These are the advantages of race, wealth, connexions, friendships, power, good health, beauty, vigour, talent, and the rest of the attributes that are either physical or externally imposed" (Cicerón, trad. de 1988, pp. 230-231 [2.10.45-46]).

$x$ "Let us then define happiness as well-being combined with virtue [. . . If then, such is the nature of happiness, its component parts must necessarily be: noble birth, numerous friends, good friends, wealth, good children, numerous children, a good old age; further, bodily excellences, such as health, beauty, strength, stature, fitness for athletic contests, a good reputation, honour, good luck, virtue" (Aristóteles, trad. en 2006, pp. 46-49 [1.5.3-4 \{1300 b\}]).

xi Por in extrema res se infiere que la acción ya ha tenido un inicio (la llegada a Cortés a tierras mejicanas) y un desarrollo (batallas entre los mexicas y los españoles, con una victoria tajante del segundo grupo) y que se está ahora en el punto del desenlace, aunque no necesariamente en el último momento.

xii "Estoy vencido, dioses eternos. En un solo día, han muerto todo el esplendor de mis fastos y la alta gloria del Méjico valeroso" (Bartrina, trad. en 1992, p. 91 [1.1]). xiii "MON. [. . .] Mientras tanto sé valiente, jtoma! (Le da un puñal) Qu esto te sirva de instrumento para mostrar tu gran corazón, $y$, antes de que el traidor te encadene como una esclava, hiere el corazón de tu hija e, inmediatamente, suicídate" (Bartrina, trad. en 1992, p. 93 [1.2]).

xiv “'Teutile y un español, si son sacrificados, podrán salvar el imperio y al padre"” (Bartrina, trad. en 1992, p. 137 [2.13]).

xv "MON. Se ven en vuestros Dioses grandes verdades. Méjico cae, es verdad; y después renace. CORO. Himeneo, tú que eres del amor el dulce ardor, el nudo

La Revista Estudios es editada por la Universidad de Costa Rica y se distribuye bajo una Licencia Creative Commons Atribución-NoComercial-CompartirIgual 3.0 Costa Rica. Para más información envíe un mensaje a revistaestudios.eeg@ucr.ac.cr. 
Dossier: La escritura en los desplazamientos: viajes, expediciones y naufragios

inmortal para esta pareja noble y real, une las almas y encadena los corazones" (Bartrina, trad. en 1992, p. 159 [3.12]). xvi "[. . .] México es dichoso. Este es el fruto de aquella libertad que unida a la prudencia sólo se subordina a aquellas leyes que yo mismo soy el primero en observar; mi pueblo goza de dicha segura y tranquilidad plena y mi poder se basa en su amor" (Reinshagen-Hernández, trad. en 1992, pp. 63-65 [1.1]).

xvii Como indica Solís, "Había [Motezuma] mostrado en diferentes ocasiones alguna inclinación a los ritos y preceptos de la fe católica, desagradando a su entendimiento los absurdos de la idolatría, y llegó a dar esperanzas de convertirse; pero siempre lo dilataba por su diabólica razón de estado [. . . ] Pero sus respuestas eran despropósitos de hombre precito [ . . . y [ . . .] rindió al demonio la eterna posesión de su espíritu, dando a la venganza y a la ferocidad las últimas cláusulas de su aliento" (Solís y Rivadeneira, versión de 1997, p. 246 [4.15]).

xviii "CORO. ¡O cielo, cuánto horror, qué día terrible, qué crimen tan vil! ¡Quién puede imaginar esta perversión! ¡Tierra, cómo toleras esto, abre las entrañas del infierno! ¡Huyan, huyan de los villanos; dioses, vengan a salvarnos, apiádense de nuestro dolor, apiádense de nuestro dolor, apiádense de nuestro dolor!" (ReinshagenHernández, trad. en 1992, p. 135 [3.5]).

xix Incluso para los mexicas, como apunta Solís, la llegada de Cortés a sus tierras se asociaba con recientes "presagios y portentos de grande horror y admiración, ordenados o permitidos por el cielo para quebrantar aquellos ánimos feroces, y hacer menos imposible a los españoles aquella grande obra que con medios tan desiguales iba disponiendo y encaminando su providencia" (Solís y Rivadeneira, versión de 1997, p. 78 [2.3]).

\footnotetext{
(c) (1)(2)

La Revista Estudios es editada por la Universidad de Costa Rica y se distribuye bajo una Licencia Creative Commons Atribución-NoComercial-CompartirIgual 3.0 Costa Rica. Para más información envíe un mensaje a revistaestudios.eeg@ucr.ac.cr.
} 